\title{
An alternative proof for the explicit equations of motion for mechanical systems with independent non-ideal constraints
}

\author{
Po-Chih Chen ${ }^{a}$, Chia-Ou Chang ${ }^{\mathrm{a}, *}$, W.T. Chang Chien ${ }^{\mathrm{b}}$ \\ a Institute of Applied Mechanics, National Taiwan University, Taipei 106, Taiwan \\ b Department of Information Management, Fooyin University, Ta-Liao, Kaohsiung Hsien, Taiwan
}

\begin{abstract}
An alternative proof for the explicit equations of motion for mechanical systems with independent non-ideal constraints is proposed in this paper. These equations are derived without the use of generalized inverse matrices and based on the decomposition of the constraint forces along the acceleration-constrained flat surface and its normal plane.

(c) 2007 Elsevier Inc. All rights reserved.
\end{abstract}

Keywords: Non-ideal constraints; Vector decomposition; Constraint forces; Ordinary differential equations of motion

\section{Introduction}

Udwadia and Kalaba investigated the mechanical systems subjected to constraints which are not necessarily independent and may be nonlinear in generalized velocity. They derived the explicit equations of motion which are free of Lagrange multipliers and in terms of generalized inverse of a rank-deficiency matrix, by using Gauss's principle [1].

Next they extended this method to the problem in which non-ideal constraints are included $[2,3]$. The characteristics of non-ideal constraint forces is that they do work under virtual displacements. If the nonholonomic ideal constraints expressed in terms of the components of generalized velocity are linear, they can be put in the Paffian form and then the subspace where the virtual displacements lie on can be determined. But this method fails when those constraints are nonlinear. Udwadia and Kalaba [4] revisited Gauss's Principle and derived the equations governing the virtual displacements for systems with constraints being nonlinear in generalized velocity. Because of this success they [5] can afford to derive the equations of motion of systems with non-ideal constraints without the use of generalized inverses. Recently, also based on the use of generalized inverse matrices, they $[6,7,8]$ had derived the general minimum principle of analytical dynamics, which encompasses Gauss's Principle, and had extended their formulation to handle the problem of singular mass matrices [9]. There are many situations where constraints can be known to be independent in advance. In these cases the equations of motion of Udwadia et al. [5] may be further reduced to simpler ones. In this brief a local

\footnotetext{
* Corresponding author.

E-mail address: changco@spring.iam.ntu.edu.tw (C.-O. Chang).
} 
coordinate space, which is composed of the subspaces tangential and normal to the acceleration-constrained surface, is established. Through the decompositions of constrained forces and accelerations into this local space, we derive the equations of motion for mechanical systems with independent constraints in a new form where the matrices involved have smaller size.

\section{Background statement}

Suppose that a mechanical system has $n$ generalized coordinates represented by the vector $\mathbf{q}=\left(q_{1}, q_{2}, \ldots\right.$, $\left.q_{n}\right)^{\mathbf{T}}$. This system is subject to $l$ non-ideal constraints, which are independent, holonomic and/or nonholonomic, such as

$$
\boldsymbol{\Phi}(\mathbf{q}, \dot{\mathbf{q}}, t)=\mathbf{0} .
$$

The equations of motion of this system can be written in the form

$$
\mathbf{M} \ddot{\mathbf{q}}=\mathbf{f}+\mathbf{f}^{\mathrm{c}} \text {, }
$$

where $\mathbf{f}$ is the vector of applied force and $\mathbf{f}^{\mathfrak{c}}$ the vector of constraint force associated with the constraints (1). The differentiation of (1) with respect to time gives

$$
\mathbf{A}(\mathbf{q}, \dot{\mathbf{q}}, t) \ddot{\mathbf{q}}=\mathbf{b}(\mathbf{q}, \dot{\mathbf{q}}, t),
$$

where $\mathbf{A}$ is a $l \times n$ matrix that has rank $l$. Let the scaled acceleration denoted by $\ddot{\mathbf{q}}_{\mathrm{s}}=\mathbf{M}^{1 / 2} \ddot{\mathbf{q}}$, the scaled applied force and constraint forces by $\mathbf{f}_{\mathrm{s}}=\mathbf{M}^{-1 / 2} \mathbf{f}$ and $\mathbf{f}_{\mathrm{s}}^{\mathrm{c}}=\mathbf{M}^{-1 / 2} \mathbf{f}^{\mathrm{c}}$, and $\mathbf{B}=\mathbf{A} \mathbf{M}^{-1 / 2}$. Then system Eqs. (2) and (3) become

$$
\ddot{\mathbf{q}}_{\mathrm{s}}=\mathbf{f}_{\mathrm{s}}+\mathbf{f}_{\mathrm{s}}^{c}, \quad \mathbf{B}\left(\mathbf{q}_{\mathrm{s}}, \dot{\mathbf{q}}_{\mathrm{s}}, t\right) \ddot{\mathbf{q}}_{\mathrm{s}}=\mathbf{b} .
$$

The virtual displacement $\mathbf{w}$ satisfies the equation $\mathbf{A}(\mathbf{q}, \dot{\mathbf{q}}, t) \mathbf{w}=\mathbf{0}[4]$. Denoting $\mathbf{v}=\mathbf{M}^{1 / 2} \mathbf{w}$, we have

$$
\mathbf{B v}=\mathbf{0} \text {. }
$$

The total constraint forces $\mathbf{f}_{\mathrm{s}}^{\mathrm{c}}$ contain the ideal and non-ideal ones. Let the non-ideal constraint force denoted by $\mathbf{C}_{\mathrm{s}}=\mathbf{M}^{-\frac{1}{2}} \mathbf{C}$. The work done by the total constraint forces under the virtual displacements is equal to the work done by the non-ideal ones as $\mathbf{V}^{\mathrm{T}} \mathbf{f}_{\mathrm{s}}^{\mathrm{c}}=\mathbf{V}^{\mathrm{T}} \mathbf{C}_{\mathrm{s}}$, where the columns of matrix $\mathbf{V}$ are the solutions of Eq. (5), and there are $n-l$ linearly independent such solutions $\mathbf{v}_{i}$, that is, $\mathbf{V}=\left[\mathbf{v}_{1}, \ldots, \mathbf{v}_{n-l}\right]$. Then the explicit equations of motion without the use of generalized inverses obtained by Udwadia and Kalaba [3] is in the form

$$
\mathbf{M} \ddot{\mathbf{q}}=\mathbf{f}+\mathbf{M}^{\frac{1}{2}} \mathbf{J} \mathbf{B}^{\mathbf{T}}(\mathbf{b}-\mathbf{A a})+\mathbf{M}^{\frac{1}{2}}\left(\mathbf{I}-\mathbf{J B}^{\mathbf{T}} \mathbf{B}\right) \mathbf{M}^{-\frac{1}{2}} \mathbf{C},
$$

where $\mathbf{J}=\left(\mathbf{B}^{\mathbf{T}} \mathbf{B}+\mathbf{V} \mathbf{V}^{\mathbf{T}}\right)^{-1}$ and $\mathbf{a}=\mathbf{M}^{-1} \mathbf{f}$.

\section{Local coordinate space}

At any instant with positions and velocities given, Eq. (4b) represents a flat surface denoted by $S_{t}$ in the scaled acceleration coordinate system. Let the columns of matrix $\mathbf{B}^{\mathbf{T}}$ be denoted by $\mathbf{b}_{1}, \mathbf{b}_{2}, \ldots, \mathbf{b}_{l}$, which are the normal vectors of $S_{t}$ and can be viewed as the basic vectors of the subspace $S_{n}$ normal to $S_{t}$. From Eq. (5) the column vectors $\mathbf{v}_{1}, \mathbf{v}_{2}, \ldots, \mathbf{v}_{n-l}$ of $\mathbf{V}$ can be considered as the basic vectors of $S_{t}$. Then the subspaces $S_{t}$ and $S_{n}$ form a local coordinate space at any time $t$ with $\mathbf{q}_{\mathrm{s}}$ and $\dot{\mathbf{q}}_{s}$ given. Any vector $\mathbf{z}$ can be decomposed along this local coordinate space as

$$
\mathbf{z}=\mathbf{B}^{\mathrm{T}} \mathbf{z}_{n}+\mathbf{V} \mathbf{z}_{t}=\left[\mathbf{B}^{\mathrm{T}}, \mathbf{V}\right]\left\{\begin{array}{l}
\mathbf{z}_{n} \\
\mathbf{z}_{t}
\end{array}\right\}=\mathbf{D}(\mathbf{z})_{\mathrm{loc}}
$$

where $\mathbf{z}_{n}$ and $\mathbf{z}_{t}$ are the components of $\mathbf{z}$ in subspaces $S_{n}$ and $S_{t}$, respectively. We can also decompose $\mathbf{z}$ into components along the scaled acceleration coordinate system, with $\mathbf{e}_{1}, \ldots, \mathbf{e}_{n}$ being the unit vectors, as $\mathbf{z}=z_{i} \mathbf{e}_{i}=\left(z_{1}, z_{2}, \ldots, z_{n}\right)^{\mathbf{T}}$. Then the components of a vector in these two different coordinate system satisfies the relation

$$
\mathbf{z}=\mathbf{D}(\mathbf{z})_{\text {loc }} .
$$


The following two identities are useful in the simplification of equations of motion.

Proposition I. Matrices $\mathbf{B}$ and $\mathbf{V}$ defined by Eqs. (4b) and (5) satisfies the following identity

$$
\mathbf{B}^{\mathbf{T}}\left(\mathbf{B B}^{\mathbf{T}}\right)^{-1} \mathbf{B}+\mathbf{V}\left(\mathbf{V}^{\mathbf{T}} \mathbf{V}\right)^{-1} \mathbf{V}^{\mathbf{T}}=\mathbf{1}
$$

Proof. For an arbitrary vector $\mathbf{z} \in \mathbf{R}^{n}$ we can express it like Eq. (7). Premultiplying both sides of Eq. (7) by $\mathbf{B}$ and using Eq. (5) give

$$
\mathbf{B} \mathbf{z}=\mathbf{B B}^{\mathbf{T}} \mathbf{z}_{n} \quad \text { or } \quad \mathbf{z}_{n}=\left(\mathbf{B B}^{\mathbf{T}}\right)^{-1} \mathbf{B} \mathbf{z} .
$$

Similarly, we can obtain

$$
\mathbf{z}_{t}=\left(\mathbf{V}^{\mathbf{T}} \mathbf{V}\right)^{-1} \mathbf{V}^{\mathbf{T}} \mathbf{z}
$$

Substituting these into Eq. (7) for $\mathbf{z}_{n}$ and $\mathbf{z}_{t}$ yields $\mathbf{z}=\left[\mathbf{B}^{\mathbf{T}}\left(\mathbf{B B}^{\mathbf{T}}\right)^{-1} \mathbf{B}+\mathbf{V}\left(\mathbf{V}^{\mathbf{T}} \mathbf{V}\right)^{-1} \mathbf{V}^{\mathbf{T}}\right] \mathbf{z}$. From this Eq. (9) comes out.

Proposition II. Matrices $\mathbf{B}$ and $\mathbf{V}$ defined by Eqs. (4b) and (5) satisfies the following identity

$$
\mathbf{D}^{-1} \mathbf{B}^{\mathbf{T}}=\left[\begin{array}{c}
\left(\mathbf{B B}^{\mathbf{T}}\right)^{-1} \mathbf{B} \\
\mathbf{0}_{(n-l) \times n}
\end{array}\right] \mathbf{B}^{\mathbf{T}}=\left[\begin{array}{c}
\mathbf{I}_{l \times l} \\
\mathbf{0}_{(n-l) \times l}
\end{array}\right]_{n \times l} .
$$

Proof. When the $i$ th basic vector $\mathbf{b}_{i}$ of matrix $\mathbf{B}^{\mathbf{T}}$ is projected into the local coordinate space, according to Eq. $(10 a, b)$, we have

$$
\left(\mathbf{b}_{i}\right)_{\mathrm{loc}}=\left\{\begin{array}{c}
\left(\mathbf{B B}^{\mathbf{T}}\right)^{-1} \mathbf{B} \mathbf{b}_{i} \\
\left(\mathbf{V}^{\mathbf{T}} \mathbf{V}\right)^{-1} \mathbf{V}^{\mathbf{T}} \mathbf{b}_{i}
\end{array}\right\}=\left\{\begin{array}{c}
\left(\mathbf{B B}^{\mathbf{T}}\right)^{-1} \mathbf{B} \mathbf{b}_{i} \\
\mathbf{0}_{(n-l) \times l}
\end{array}\right\}=\left[\begin{array}{c}
\left(\mathbf{B B}^{\mathbf{T}}\right)^{-1} \mathbf{B} \\
\mathbf{0}_{(n-l) \times n}
\end{array}\right]_{n \times n} \mathbf{b}_{i}
$$

since $\mathbf{b}_{i} \in S_{n}, \mathbf{V}^{\mathbf{T}} \mathbf{b}_{i}=\mathbf{0}$.

Let the local-coordinate representation of matrix $\mathbf{B}^{\mathbf{T}}$ be denotes by $\left(\mathbf{B}^{\mathbf{T}}\right)_{\mathrm{loc}}=\left[\left(\mathbf{b}_{1}\right)_{\mathrm{loc}}, \ldots,\left(\mathbf{b}_{l}\right)_{\mathrm{loc}}\right]$. Then the use of Eq. (12) gives

$$
\begin{aligned}
\left(\mathbf{B}^{\mathbf{T}}\right)_{\mathrm{loc}} & =\left[\left[\begin{array}{c}
\left(\mathbf{B B}^{\mathbf{T}}\right)^{-1} \mathbf{B} \\
\mathbf{0}_{(\mathbf{n}-l) \times n}
\end{array}\right] \mathbf{b}_{1}, \ldots,\left[\begin{array}{c}
\left(\mathbf{B B}^{\mathbf{T}}\right)^{-1} \mathbf{B} \\
\mathbf{0}_{(\mathbf{n}-l) \times n}
\end{array}\right] \mathbf{b}_{l}\right]=\left[\begin{array}{c}
\left(\mathbf{B B}^{\mathbf{T}}\right)^{-1} \mathbf{B} \\
\mathbf{0}_{(\mathbf{n}-l) \times n}
\end{array}\right]\left[\mathbf{b}_{1}, \ldots, \mathbf{b}_{l}\right] \\
& =\left[\begin{array}{c}
\left(\mathbf{B B}^{\mathbf{T}}\right)^{-1} \mathbf{B} \\
\mathbf{0}_{(\mathbf{n}-l) \times n}
\end{array}\right] \mathbf{B}^{\mathbf{T}}=\left[\begin{array}{c}
\mathbf{I}_{l \times l} \\
\mathbf{0}_{(\mathbf{n}-l) \times l}
\end{array}\right]_{n \times l}
\end{aligned}
$$

In other way, from Eq. (8) we have $\left(\mathbf{b}_{i}\right)_{\operatorname{loc}}=\mathbf{D}^{-1} \mathbf{b}_{i}$. Then

$$
\left(\mathbf{B}^{\mathbf{T}}\right)_{\mathrm{loc}}=\left[\left(\mathbf{b}_{1}\right)_{\mathrm{loc}}, \ldots,\left(\mathbf{b}_{l}\right)_{\mathrm{loc}}\right]=\left[\mathbf{D}^{-1} \mathbf{b}_{1}, \ldots, \mathbf{D}^{-1} \mathbf{b}_{l}\right]=\mathbf{D}^{-1}\left[\mathbf{b}_{1}, \ldots, \mathbf{b}_{l}\right]=\mathbf{D}^{-1} \mathbf{B}^{\mathbf{T}}
$$

The comparison of (13b) with (13a) gives Eq. (11).

\section{Equations of motion}

Proposition III. The equations of motion (6) is equivalent to

$$
\mathbf{M} \ddot{\mathbf{q}}=\mathbf{f}+\mathbf{M}^{\mathbf{1} / \mathbf{2}} \mathbf{B}^{\mathrm{T}}\left(\mathbf{B} \mathbf{B}^{\mathbf{T}}\right)^{-1}(\mathbf{b}-\mathbf{A a})+\mathbf{M}^{1 / 2} \mathbf{V}\left(\mathbf{V}^{\mathbf{T}} \mathbf{V}\right)^{-1} \mathbf{V}^{\mathrm{T}} \mathbf{M}^{-\mathbf{1} / \mathbf{2}} \mathbf{C} .
$$

Proof. We first simplify the matrix $\mathbf{J B}^{\mathbf{T}}$ appearing in Eq. (6).

$$
\mathbf{J B}^{\mathbf{T}}=\left(\mathbf{B}^{\mathbf{T}} \mathbf{B}+\mathbf{V} \mathbf{V}^{\mathbf{T}}\right)^{-1} \mathbf{B}^{\mathbf{T}}=\left[\left[\mathbf{B}^{\mathbf{T}}, \mathbf{V}\right]\left[\begin{array}{c}
\mathbf{B} \\
\mathbf{V}^{\mathbf{T}}
\end{array}\right]\right]^{-1} \mathbf{B}^{\mathbf{T}}=\left[\begin{array}{c}
\mathbf{B} \\
\mathbf{V}^{\mathbf{T}}
\end{array}\right]^{-1}\left[\mathbf{B}^{\mathbf{T}}, \mathbf{V}\right]^{-1} \mathbf{B}^{\mathbf{T}}
$$


Using Eq. (11), the above equation reduces to

$$
\mathbf{J B}^{\mathbf{T}}=\left[\begin{array}{c}
\mathbf{B} \\
\mathbf{V}^{\mathbf{T}}
\end{array}\right]^{-1}\left[\begin{array}{c}
\mathbf{I}_{l \times l} \\
\mathbf{0}_{(n-l) \times l}
\end{array}\right]_{n \times l} .
$$

For simplicity let $\mathbf{K}_{n \times l}=\mathbf{J B}^{\mathbf{T}}$, where $\mathbf{K}=\left[\mathbf{k}_{1}, \ldots, \mathbf{k}_{l}\right]$ and $\mathbf{k}_{i}$ is an $n \times 1$ vector. The inverse of Eq. (15) gives

$$
\left[\begin{array}{c}
\mathbf{B} \\
\mathbf{V}^{\mathbf{T}}
\end{array}\right] \mathbf{K}_{n \times l}=\left[\begin{array}{c}
\mathbf{I}_{l \times l} \\
\mathbf{0}_{(n-l) \times l}
\end{array}\right]_{n \times l} .
$$

The second set of Eq. (16) is $\mathbf{V}^{\mathrm{T}} \mathbf{K}=\mathbf{0}_{(n-l) \times l}$, which means that each column vector of $\mathbf{K}$ lies on the subspace $S_{n}$. Hence the projection of the $i$ th column vector of matrix $\mathbf{K}$ into the local coordinate space gives $\mathbf{k}_{i}=\mathbf{B}^{\mathrm{T}} \mathbf{k}_{i n}$. Then matrix $\mathbf{K}$ can be put in the form

$$
\mathbf{K}_{n \times l}=\left[\mathbf{B}^{\mathbf{T}} \mathbf{k}_{1 n}, \mathbf{B}^{\mathbf{T}} \mathbf{k}_{2 n}, \ldots, \mathbf{B}^{\mathbf{T}} \mathbf{k}_{l n}\right]=\mathbf{B}^{\mathbf{T}} \mathscr{K}_{l \times l} .
$$

Substituting (17) into the first set of Eq. (16) yields

$$
\mathbf{B B}^{\mathbf{T}} \mathscr{K}_{l \times l}=\mathbf{I}_{l \times l} \quad \text { or } \quad \mathscr{K}_{l \times l}=\left(\mathbf{B B}^{\mathbf{T}}\right)^{-1} .
$$

Therefore,

$$
\mathbf{J B}^{\mathbf{T}}=\mathbf{K}=\mathbf{B}^{\mathbf{T}}\left(\mathbf{B B}^{\mathbf{T}}\right)^{-1} .
$$

The second term in the right-hand side of Eq. (6), which is the ideal constraint force, now becomes

$$
\mathbf{M}^{1 / 2} \mathbf{J B}^{\mathbf{T}}(\mathbf{b}-\mathbf{A a})=\mathbf{M}^{1 / 2} \mathbf{B}^{\mathbf{T}}\left(\mathbf{B B}^{\mathbf{T}}\right)^{-1}(\mathbf{b}-\mathbf{A a}) .
$$

With the use of Eqs. (19) and (9) the third term in the right-hand side of Eq. (6), representing the non-ideal constraint force, becomes

$$
\mathbf{M}^{1 / 2}\left(\mathbf{I}-\mathbf{J B}^{\mathbf{T}} \mathbf{B}\right) \mathbf{M}^{-1 / 2} \mathbf{C}=\mathbf{M}^{1 / 2}\left[\mathbf{I}-\mathbf{B}^{\mathbf{T}}\left(\mathbf{B} \mathbf{B}^{\mathbf{T}}\right)^{-1} \mathbf{B}\right] \mathbf{M}^{-1 / 2} \mathbf{C}=\mathbf{M}^{1 / 2} \mathbf{V}\left(\mathbf{V}^{\mathbf{T}} \mathbf{V}\right)^{-1} \mathbf{V}^{\mathbf{T}} \mathbf{M}^{-1 / 2} \mathbf{C} .
$$

So Eq. (14) is indeed equivalent to Eq. (6) for the case where constraints are independent. The expressions for the ideal and non-ideal constraint forces in Eq. (14) carry geometrical meanings that the ideal constraint force is perpendicular to the acceleration-constrained flat surface, while the non-ideal one lies on that surface.

The second term in the right-hand side of Eq. (6) need to calculate the inverse matrix $\mathbf{J}=\left(\mathbf{B}^{\mathbf{T}} \mathbf{B}+\mathbf{V V}^{\mathbf{T}}\right)^{-1}$, which is a $n \times n$ one, while the second term in the right-hand side of Eq. (14) have to calculate matrix $\left(\mathbf{B B}^{\mathbf{T}}\right)^{-1}$, which is a $l \times l$ one and smaller in size than $\mathbf{J}$. The non-ideal constraint force, i.e., the third term in the righthand side of Eq. (6) also require to evaluate the $n \times n$ matrix $\mathbf{J}$, but the corresponding one in Eq. (14) has to calculate the $(n-l) \times(n-l)$ matrix $\left(\mathbf{V}^{\mathbf{T}} \mathbf{V}\right)^{-1}$, which is also smaller than $\mathbf{J}$. So, when constraints are independent, Eq. (6) can be simplified to a new set of equations of motion in the form of Eq. (14), which involves inversing smaller matrices and will be more convenient for analysis. Eq. (14) can also be obtained easily from the general equation given by Udwadia et al. [5] by the use of generalized inverse of matrices to justify the existence of Eq. (14), while the presented method provides additional insights into the physics and geometry of constrained forces.

\section{Conclusions}

For the situation where the constraint equations can be justified to be independent, then the equations of motion appeared recently in the literature for mechanical systems with ideal and/or non-ideal constraints, which may not be independent, can be reduced to the ones that involve the inversing of smaller matrices. The simplification is accomplished by introducing a local coordinate space, which is composed of the acceleration-constrained flat surface and its normal surface.

\section{References}

[1] F.E. Udwadia, R.E. Kalaba, A new perspective on Constrainted Motion, Proc. R. Soc. London, Se. A 439 (1992) $407-410$.

[2] F.E. Udwadia, R.E. Kalaba, Explicit equations of motion for mechanical systems with nonideal constraints, ASME J. Appl. Mech. 68 (2001) 462-467. 
[3] F.E. Udwadia, R.E. Kalaba, What is the general form of the explicit equations of motion for constrained mechanical systems? ASME J. Appl. Mech. 69 (2002) 335-339.

[4] F.E. Udwadia, R.E. Kalaba, Analytical Dynamics, Cambridge University Press, 1996.

[5] F.E. Udwadia, R.E. Kalaba, P. Phohomsiri, Mechanical systems with nonideal constraints: explicit equations without the use of generalized inverses, ASME J. Appl. Mech. 71 (2004) 615-621.

[6] F.E. Udwadia, New general principle of mechanics and its application to general nonideal nonholonomic systems, J. Eng. Mechan. 13 (4) (2005) 444-450.

[7] F.E. Udwadia, Equations of motion for constrained multibody systems and their control, J. Optimiz. Theory Appl. 127 (3) (2005) 627638.

[8] F.E. Udwadia, On constrained motion, Appl. Math. Comput. 164 (2005) 313-320.

[9] F.E. Udwadia, P. Phohomsiri, Explicit equations of motion for constrained mechanical systems with singular mass matrices and applications to multi-body dynamics, Proc. R. Soc., Se. A (March) (2006) 1-21. 